\title{
Statistics of Bicoherence and Biphase
}

\author{
Steve Elgar and Gloria Sebert
}

Electrical and Computer Engineering Department, Washington State University, Pullman

\begin{abstract}
Statistics of estimates of bicoherence and biphase were obtained from numerical simulations of nonlinear random (harmonic) processes with known true bicoherence and biphase. Expressions for the bias, variance, and probability distributions of estimates of bicoherence and biphase as functions of the true bicoherence and number of degrees of freedom (dof) used in the estimates are presented. The probability distributions are consistent with theoretical distributions derived for the limit of infinite dof and are used to construct confidence limits on estimates of bicoherence and biphase. Maximum likelihood estimates of true values of bicoherence and biphase given observed values are also presented.
\end{abstract}

\section{INTRODUCTION}

Bispectral analysis has been used to study nonlinear interactions in a variety of ocean processes, including surface gravity waves in intermediate water depths [Hasselman et al., 1963], perturbations from the mean profiles of temperature, salinity and sound velocity [Roden and Bendiner, 1973], internal waves [Neshyba and Sobey, 1975; McComas and Briscoe, 1980], shoaling surface gravity waves [Elgar and Guza, 1985, 1986; Doering and Bowen, 1987], and temperature fluctuations [Müller, 1987]. A wide range of phenomena in other physical systems has also been investigated with the bispectrum (see Nikias and Raghuveer [1987] for a recent review). In most of these studies the bispectrum was used to determine whether or not the process under investigation was consistent with linear dynamics. Specifically, nonlinear interactions are associated with nonzero values of the bicoherence, defined here as [Haubrich, 1965; Kim and Powers, 1979]

$$
b^{2}\left(\omega_{1}, \omega_{2}\right)=\frac{\left|B\left(\omega_{1}, \omega_{2}\right)\right|^{2}}{E\left[\left|A\left(\omega_{1}\right) A\left(\omega_{2}\right)\right|^{2}\right] E\left[\left|A\left(\omega_{1}+\omega_{2}\right)\right|^{2}\right]}
$$

where $B$ is the bispectrum,

$$
B\left(\omega_{1}, \omega_{2}\right)=E\left[A\left(\omega_{1}\right) A\left(\omega_{2}\right) A^{*}\left(\omega_{1}+\omega_{2}\right)\right]
$$

$A(\omega)$ is the complex Fourier coefficient of the time series at radian frequency $\omega$ and $E[\quad]$ is the expected, or average, value. Alternately, the bispectrum can be calculated as the Fourier transform of the third-order correlation function of the time series [Hasselman et al., 1963]. For a finite length time series, even a truly linear (e.g., Gaussian) process will have nonzero bicoherence. Haubrich [1965] shows that for a Gaussian process the bicoherence (whose true value equals zero) is approximately chi-square $\left(\chi^{2}\right)$ distributed in the limit of large degrees of freedom (dof), and thus significance levels for zero bicoherence as a function of dof can be calculated. Elgar and Guza [1988] demonstrated that estimates of bicoherence from a Gaussian process are also approximately $\chi^{2}$ distributed for low values of dof $($ dof $=32)$ and are not sensitive to smoothing procedures used to increase dof.

Although the significance levels for zero bicoherence can

Copyright 1989 by the American Geophysical Union.

Paper number 89JC01034.

0148-0227/89/89JC-01034\$05.00 be used to statistically detect the presence or absence of nonlinear interactions, the statistics of estimates of bicoherence and biphase, $\beta\left(\omega_{1}, \omega_{2}\right)$, the phase of $B\left(\omega_{1}, \omega_{2}\right)$, for the case of nonzero true bicoherence have not previously been reported. The purpose of this study is to present such statistics. Brillinger [1965], Rosenblatt and Van Ness [1965], Brillinger and Rosenblatt [1967a, b], and others [see Nikias and Raghuveer, 1987] give some of the statistical properties of higher-order spectra, including asymptotic distributions of the real and imaginary parts of the bispectrum. Haubrich [1965], Hinich and Clay [1968], Kim and Powers [1979], Hinich [1982], and Ashley et al. [1986] discuss the estimation of bicoherence.

For the present study, harmonic random processes with true values of bicoherence $\left(b^{2}\right)$ between 0.1 and 1.0 were numerically simulated (section 2 ), and the statistics of estimates of bicoherence $\left(\hat{b}^{2}\right)$ and biphase $(\hat{\beta})$ obtained from the simulated time series were calculated (section 3 ). The probability distributions of $\hat{b}^{2}$ and $\hat{\beta}$, including the bias of $\hat{b}^{2}$ and the variances and confidence limits of $\hat{b}^{2}$ and $\hat{\beta}$, were determined as functions of $b^{2}$ and dof (sections 3.1 and 3.2). These values are useful for experimental design. On the other hand, once an experiment has been conducted, maximum likelihood techniques may be used to estimate true values of $b^{2}$ and $\beta$ based on the observed values. Maximum likelihood estimates of bicoherence are presented in section 3.3. These results are then briefly applied to estimates of the bicoherence and biphase of narrow-band surface gravity waves observed in $9 \mathrm{~m}$ water depth (section 3.4). Conclusions follow in section 4 .

\section{Simulation Procedure}

Time series consisting of triads of sinusoids (with frequencies $\omega_{i}, \omega_{j}$, and $\omega_{i+j}$ ) were numerically simulated on the CRAY XMP at the San Diego Supercomputer Center. By adjusting the amplitudes of the component sinsuoids, some of whose phases were chosen from a uniform random distribution $(0-2 \pi)$, the true bicoherence value of the triad could be varied. For example, consider the case of a single triad:

$$
\begin{gathered}
x(t)=\cos \left(\omega_{1} t+\phi_{1}\right)+\cos \left(\omega_{2} t+\phi_{2}\right)+a \cos \left(\omega_{3} t+\phi_{3}\right) \\
+\cos \left(\omega_{3} t+\phi_{1}+\phi_{2}\right)
\end{gathered}
$$

where $\omega_{3}=\omega_{1}+\omega_{2}, \phi_{1}, \phi_{2}$, and $\phi_{3}$ are random phases, and $a$ is an adjustable parameter. As $a$ increases from 0 to $\infty$, 
$b^{2}\left(\omega_{1}, \omega_{2}\right)$ decreases from 1.0 to 0.0 . For the example given in (3), if $a=0$, then $\beta\left(\omega_{1}, \omega_{2}\right)=0$. Time series consisting of the following triads were simulated: a single triad, $\left(\omega_{1}, \omega_{2}\right.$, $\left.\omega_{3}\right)$; two triads with the same sum frequency, $\left(\omega_{1}, \omega_{5}, \omega_{6}\right)$ and $\left(\omega_{3}, \omega_{3}, \omega_{6}\right)$; and two triads with a common frequency other than the sum frequency, $\left(\omega_{1}, \omega_{3}, \omega_{4}\right)$ and $\left(\omega_{3}, \omega_{5}, \omega_{8}\right)$. True values of $\beta=0$ and $\beta=\pi / 4$ were used. For each case the true bicoherence of each triad could be adjusted. The statistics of estimates of bicoherence and biphase reported here were found to depend only on true values of $b^{2}$ and dof; they did not depend on the number of triads, nor on how the triads were interrelated, nor on $\beta$. Additional time series were simulated with Gaussian-distributed Fourier coefficients rather than uniformly distributed random phases [Elgar et al., 1985, and references therein]. The resulting statistics of $\hat{b}^{2}$ and $\hat{\beta}$ were essentially identical to those of the random phase simulations.

Each simulation consisted of generating many realizations of 65,536-point time series, each of which was subdivided into short sections of 256 points. The short sections were not tapered. By ensemble averaging the bispectrum over all 256 of the short records, estimates of bicoherence and biphase with 512 dof were produced from each 65,536-point time series. Each 65,536-point record was also subdivided into 16 groups of 16 short records, each group producing estimates with 32 dof. Estimates with dof between 32 and 512 were obtained in a similar manner. One thousand of the 65,536point time series were generated, and thus 1000 (dof $=512$ ) to 16,000 (dof $=32$ ) bicoherence and biphase estimates were produced for each true value of bicoherence. Results from simulations using 100 realizations were indistinguishable from those where averages of 1000 realizations were used.

\section{Simulation Results}

\subsection{Bicoherence}

As shown below, $b^{2}$ is approximately chi-square distributed and thus will have a positive bias, similar to the ordinary coherence between two time series [Jenkins and Watts, 1968; Beningus, 1969]. Following Beningus [1969], the bias was empirically found to be approximately given by

$$
\operatorname{Bias}\left[\hat{b}^{2}\right]=(2 / \text { dof })\left(1-b^{2}\right)^{2}
$$

Equation (4) is similar to the corresponding expression for coherence [Beningus, 1969] and is compared to the observed bias in Figure 1. Guided by expressions based on somewhat heuristic theoretical arguments [Hinich and Clay, 1968; Kim and Powers, 1979], the variance of $b^{2}$ was empirically found to be approximately

$$
\operatorname{Var}\left[b^{2}\right]=\left(4 b^{2} / \mathrm{dof}\right)\left(1-b^{2}\right)^{3}
$$

Equation (5) is similar to the analogous approximate theoretical equation for coherence [Jenkins and Watts, 1968], and the agreement between (5) and the simulated data is excellent, as shown in Figure 2.

Haubrich [1965] suggests that for a process with $b^{2}=0.0$, $b^{2}$ will asymptotically (large dof) approach a chi-square distribution with parameter $\nu=2$. Hinich [1982] shows that bicoherence is approximately asymptotically distributed as a noncentral chi-square random variable. The noncentral chisquare distribution can be approximated by an $\alpha \chi^{2}$ distribution [Abramowitz and Stegun, 1972], given by

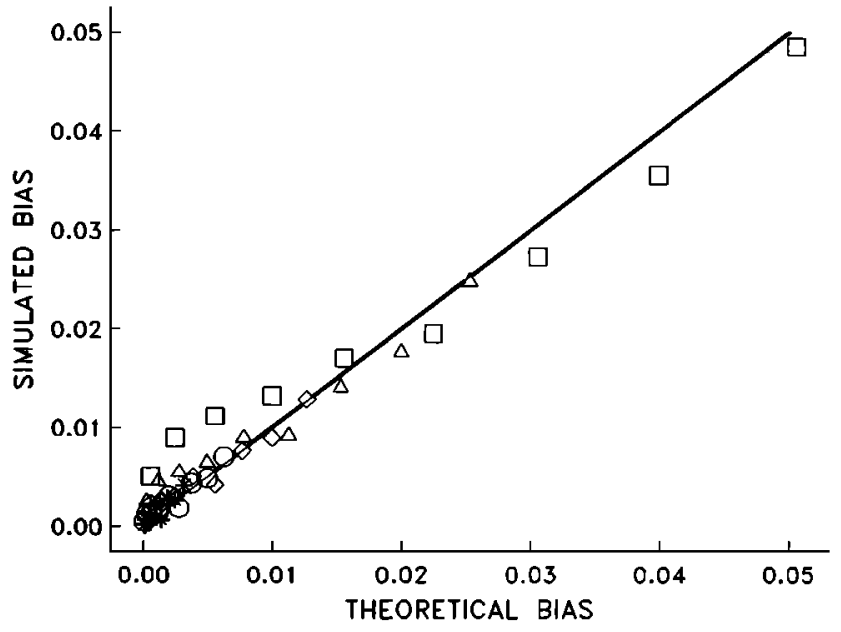

Fig. 1. Bias of bicoherence observed in simulated data versus the theoretical bias (equation (4)). Squares, dof $=32$; triangles, dof $=64$; diamonds, dof $=128$; circles, dof $=256$; and asterisks, dof $=$ 512. The bicoherence, $b^{2}=0.1,0.2, \cdots, 0.9$ for each dof.

$$
f_{\chi^{2}}\left(\frac{b^{2}}{\alpha}\right)=\frac{\left(b^{2}\right)^{(\nu / 2)-1} e^{-b^{2} / 2}}{2^{(\nu / 2)} \Gamma(\nu / 2)}
$$

where $\Gamma($ ) is the gamma function. The mean and variance of an $\alpha \chi^{2}$ distributed random variable are $\alpha \nu$ and $2 \alpha^{2} \nu$, respectively. Thus the parameters $\alpha$ and $\nu$ can be determined from the bicoherence as

$$
\alpha=\frac{E\left[b^{2}\right]}{\nu} \quad \nu=\frac{2 E^{2}\left[b^{2}\right]}{\operatorname{Var}\left[b^{2}\right]}
$$

Combining (5) and (7), and using the biased value of bicoherence,

$$
\nu=\frac{(\text { dof }) b^{2}}{2\left(1-b^{2}\right)^{3}}
$$

As $b^{2} \rightarrow 1$ and/or dof increases, the parameter $\nu$ increases, and $f_{\chi^{2}}\left(b^{2} / \alpha\right)$ approaches a Gaussian distribution. Probability

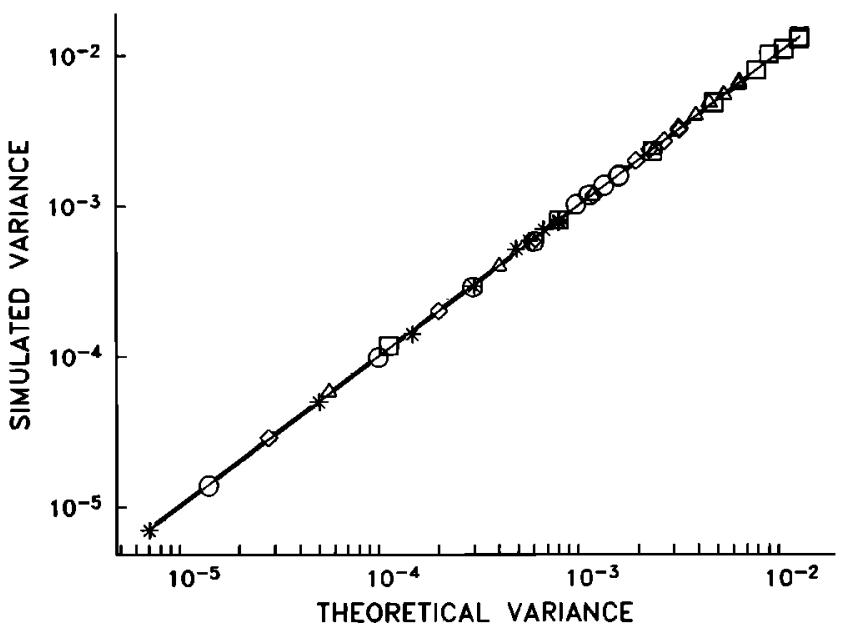

Fig. 2. Variance of bicoherence observed in simulated data versus the theoretical variance (equation (5)). Each symbol represents different dof, as described in the caption to Figure 1. 


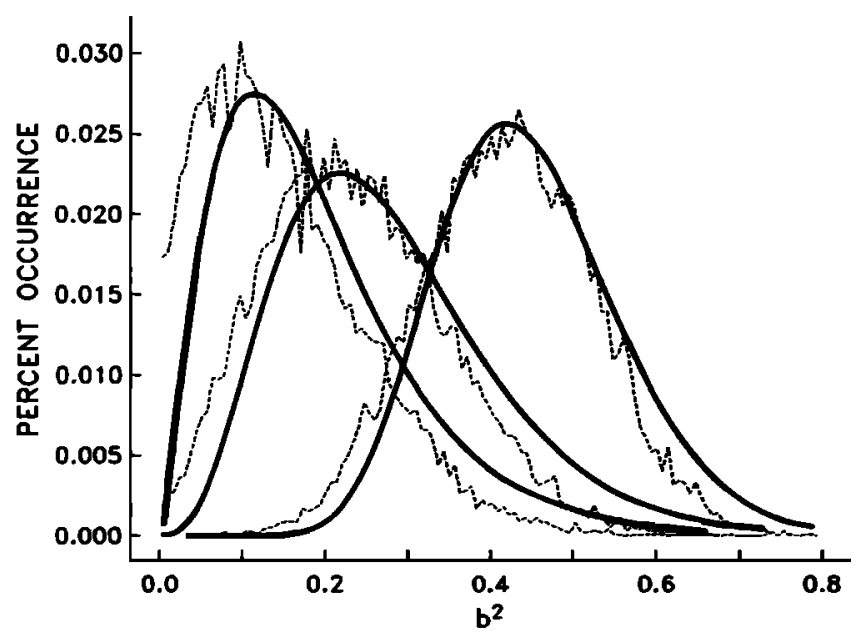

Fig. 3. Probability distribution of $\boldsymbol{b}^{2}$ observed in the simulated data for dof $=32$ (dashed curve) and theoretical $\alpha \chi^{2}$ distribution (solid curve). From left to right, $b^{2}=0.1,0.2$, and 0.4 . The bin width is $\Delta\left(b^{2}\right)=0.0067$.

distributions of the simulated data are compared to $\alpha \chi^{2}$ distributions in Figure 3, with $\alpha$ and $\nu$ obtained from (7) and (8), respectively. According to a $\chi^{2}$ goodness of fit test, the theoretical and observed distributions with 32 dof and $b^{2} \leq$ 0.4 do not differ significantly at the $95 \%$ confidence level. For 32 dof the probability distributions of the simulated data are slightly offset toward lower values of $b^{2}$ relative to the $\alpha \chi^{2}$ distributions, with the amount of offset decreasing with increasing $b^{2}$ (Figure 3). For higher values of dof the offset is virtually zero, and, consequently, no attempt was made to account for it in the probability distributions with 32 dof shown in Figure 3. The scatter in the probability distributions of the simulated data shown in Figure 3 is due to finite record lengths and finite bin width. There is even better agreement between simulated data and $\alpha \chi^{2}$ distributions as $b^{2} \rightarrow 1$ and/or dof $\rightarrow \infty$.

Assuming $\hat{b}^{2}$ is $\alpha \chi^{2}$ distributed with $\alpha$ and $\nu$ given by (7) and (8), respectively, confidence limits for $b^{2}$ can be constructed as functions of $b^{2}$ and dof. The $90 \%$ confidence limits are compared to the corresponding values observed in the simulations in Figure 4.

\subsection{Biphase}

Similar to the phase of the cross spectrum [Jenkins and Watts, 1968], the biphase is approximately Gaussian distributed, unbiased, and has variance (in units of radians)

$$
\operatorname{Var}[\hat{\beta}]=\left(\frac{1}{\operatorname{dof}}\right)\left(\frac{1}{b^{2}}-1\right)
$$

The theoretical variance and confidence limits (calculated from a Gaussian distribution with variance given by (9)) for $\hat{\beta}$ are compared to simulated values in Figures 5 and 6 , respectively.

\subsection{Maximum Likelihood Estimation}

The results of the previous sections describe the statistics of bicoherence given the true value, $b^{2}$, thus enabling the design of experiments to measure bicoherence. On the other

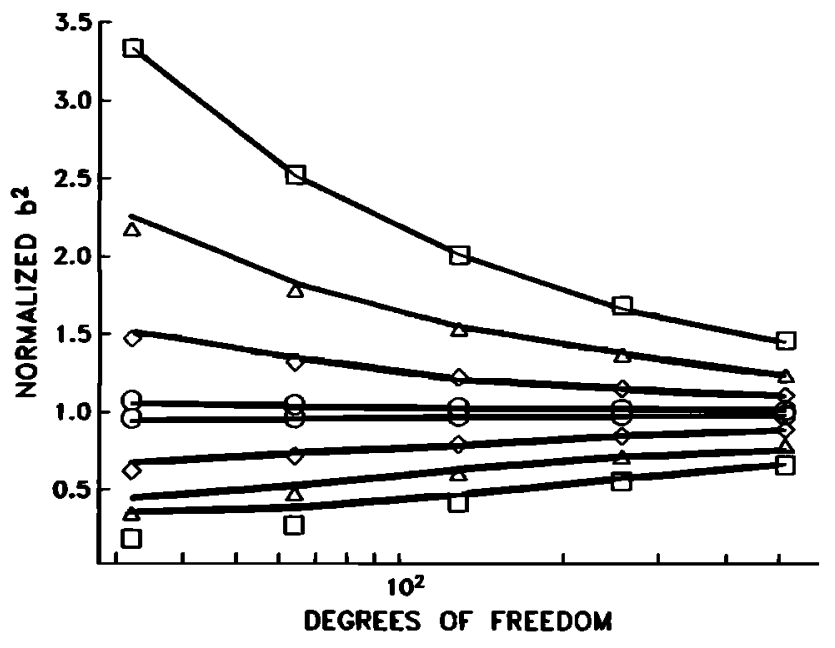

Fig. 4. The $90 \%$ confidence limits for bicoherence. The values shown have been normalized by the corresponding true value of bicoherence. Thus $90 \%$ of the time, $\hat{b}^{2}$ will be in the range given by the appropriate ordinate values times $b^{2}$. Solid curves are theoretical values, and symbols are values observed in the simulated data. Squares, $b^{2}=0.1 ;$ triangles, $b^{2}=0.2$; diamonds, $b^{2}=0.4$; and circles, $b^{2}=0.8$.

hand, it is often the case that $b^{2}$ must be estimated from a limited set of data. In this section maximum likelihood estimates [Jenkins and Watts, 1968] of the true value of bicoherence given an observed value, $b^{2}$, with finite dof are presented. Freilich and Pawka [1987] present a similar analysis for estimates of the cross spectrum, with results analogous to those reported here. Maximum likelihood estimation consists of determining parameters of the underlying probability distribution function (pdf) from samples of the distribution. The pdf is recast as a function of the parameters to be determined, and those values of the parameters that maximize this likelihood function are considered the maximum likely estimates (mle) of the true parameters of the underlying distribution given the particular samples at hand. For the case of bicoherence the likelihood function, $L\left(b^{2}\right)$, is given by the $\alpha \chi^{2}$ distribution (equation (6)) and is shown as

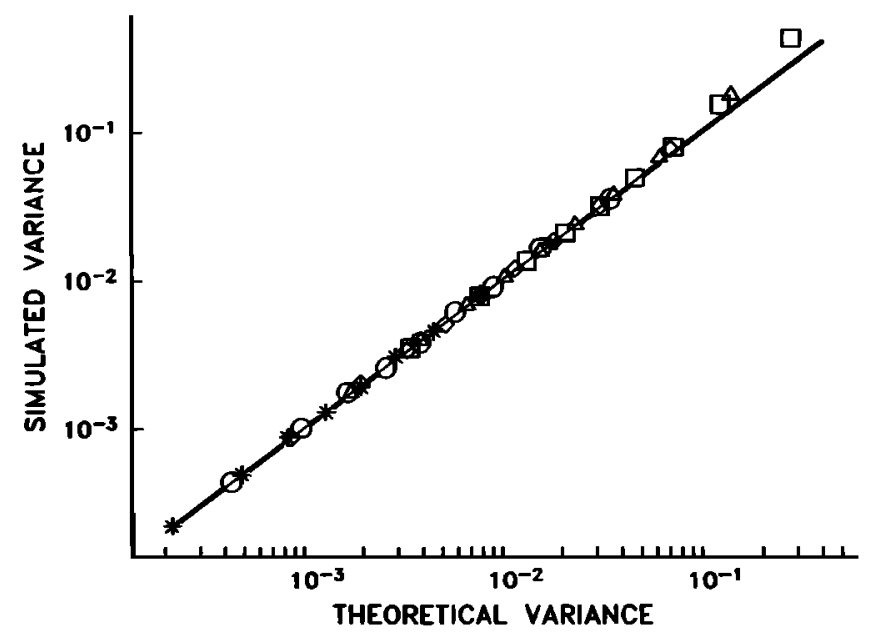

Fig. 5. Variance of biphase observed in simulated data versus the theoretical variance (equation (9)). The units are radians squared. Each symbol represents different dof, as described in the caption to Figure 1. 


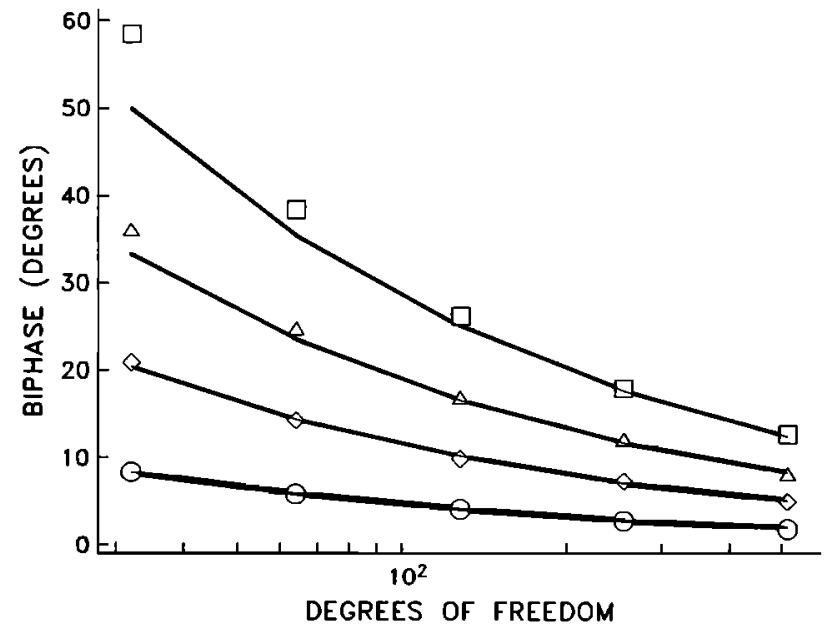

Fig. 6. The $90 \%$ confidence limits for biphase. Solid curves are theoretical values, and symbols are values observed in the simulated data. Each symbol represents different $b^{2}$ as described in the caption to Figure 4.

a function of dof and $b^{2}$ in Figure 7. For large values of $b^{2}$ and/or dof, $L\left(b^{2}\right)$ is symmetrical about its maximum. However, for small $b^{2}$ and/or dof, $L\left(b^{2}\right)$ has long tails extending toward high values of $b^{2}$ (Figure 7). In order to better account for the asymmetrical shape of the likelihood function, Jenkins and Watts [1968] suggest using the mean of the likelihood function (mele) as an estimate of the true parameter. Both mle and mele estimates of bicoherence are listed in Table 1. As $L\left(b^{2}\right)$ becomes more symmetrical, $b_{\text {mele }}^{2} \rightarrow$ $b_{\text {mle }}^{2} \rightarrow b^{2}$ (Table 1 and Figure 8), although the likelihood estimates of $b^{2}$ are always greater than the corresponding measured values (Figure 8). This can be understood by considering the $\chi^{2}$ pdfs shown in Figure 9. It is more likely

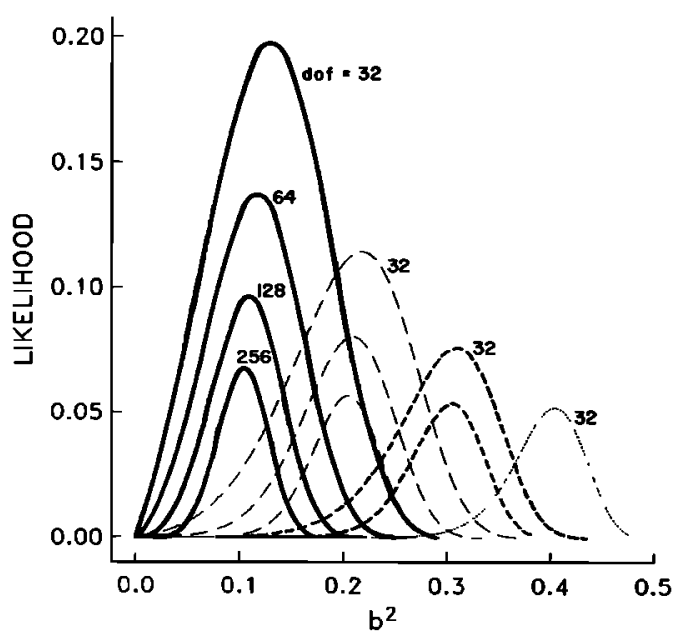

Fig. 7. Likelihood functions $L\left(b^{2}\right)$ for various measured bicoherence and dof values. Solid curves, unbiased $b^{2}=0.1$; longest dashes, $b^{2}=0.2$; medium length dashes, $b^{2}=0.3$; and shortest dashes, $\hat{b}^{2}=0.4$. For each $b^{2}$ the outermost curve has 32 dof, and the dof increase by factors of 2 for each succeeding inner curve. The number of such curves decreases for increasing $b^{2}$. Unbiased values are obtained by subtracting the bias (equation (4)) from the observed value, where the observed value of bicoherence is used on the right-hand side of (4). Replacing $b^{2}$ with $b_{\text {mele }}^{2}$ in (4) results in negligible differences in the bias.
TABLE 1. Parameters for the Likelihood Functions

\begin{tabular}{lrcccc}
\hline$b^{2}$ & dof & $b_{\text {mle }}^{2}$ & $b_{\text {mele }}^{2}$ & s.d. & $(7.5: 1)$ \\
\hline 0.1 & 32 & 0.129 & 0.146 & 0.047 & $(0.018,0.237)$ \\
0.1 & 64 & 0.116 & 0.125 & 0.041 & $(0.026,0.201)$ \\
0.1 & 128 & 0.108 & 0.113 & 0.032 & $(0.039,0.172)$ \\
0.1 & 256 & 0.104 & 0.107 & 0.024 & $(0.054,0.151)$ \\
0.2 & 32 & 0.217 & 0.223 & 0.054 & $(0.078,0.314)$ \\
0.2 & 64 & 0.209 & 0.212 & 0.042 & $(0.109,0.284)$ \\
0.2 & 128 & 0.204 & 0.206 & 0.031 & $(0.135,0.261)$ \\
0.3 & 32 & 0.309 & 0.310 & 0.047 & $(0.191,0.387)$ \\
0.3 & 64 & 0.304 & 0.305 & 0.037 & $(0.224,0.364)$ \\
0.4 & 32 & 0.403 & 0.404 & 0.045 & $(0.323,0.462)$ \\
\hline
\end{tabular}

The parameter $b^{2}$ is the unbiased observed bicoherence, dof is the degrees of freedom associated with the measurement, $b_{\text {mle }}^{2}$ is the maximum of the likelihood function $(L), b_{\text {mele }}^{2}$ is the mean of $L$, s.d. is the standard deviation of $L$, and $(7.5: 1)$ indicates values of $b^{2}$ corresponding to likelihood values down by a factor of 7.5 from the maximum of $L$ (if $L$ is Gaussian, these values correspond to $95 \%$ confidence limits).

that an observed value comes from the center of the distribution with high $\nu$ (i.e., high $b_{2}$ ) than from the upper tail of a distribution with lower $\nu$. Table 1 and Figure 8 both show that except for small $\hat{b}^{2}$ and/or dof, $b_{\text {mele }}^{2}$ does not differ substantially from $\hat{b}^{2}$.

$L\left(b^{2}\right)$ can also be used to calculate confidence intervals for the estimated true values of bicoherence. The likelihood functions presented here (Figure 7) are approximately Gaussian, and thus the mele and the variance of $L\left(b^{2}\right)$ determine the entire likelihood function [see Jenkins and Watts, 1968]. The standard deviations of $L\left(b^{2}\right)$ and the $b^{2}$ values whose likelihoods are down by a factor of 7.5 from the mle $(95 \%$ confidence limits on $b^{2}$ if $L\left(b^{2}\right)$ is Gaussian) are presented in Table 1. From the table it can be seen that the standard deviation of $L\left(b^{2}\right)$ is nearly independent of $b^{2}$ and depends primarily on dof.

Since the distribution of biphase is approximately Gaus$\operatorname{sian}, \beta_{\text {mele }} \approx \beta_{\text {mle }} \approx \hat{\beta}$.

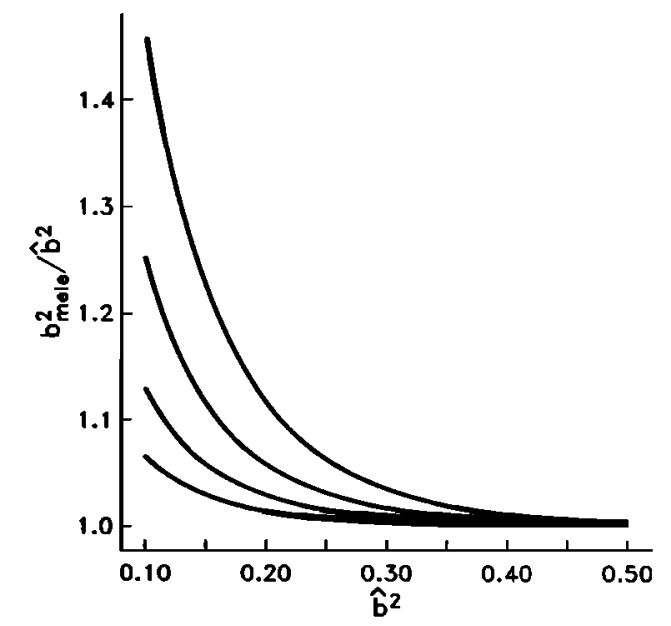

Fig. 8. Ratio of $b_{\text {mele }}^{2}$ to $b^{2}$ versus $b^{2}$. Each curve is for different dof, increasing by factors of 2 from dof $=32$ (top curve) to dof $=256$ (bottom curve). 


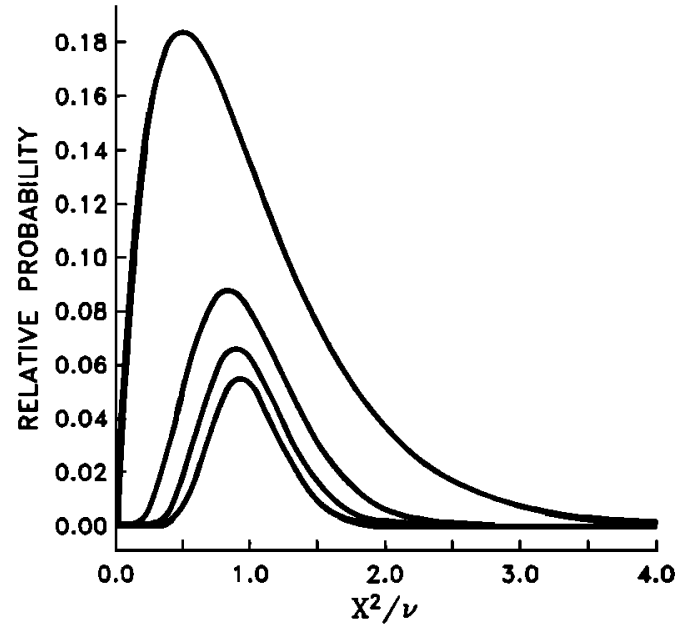

Fig. 9. Chi-square probability distribution functions. From outermost to innermost curves, $\nu=4,12,20$, and 28 , respectively. The abscissa has been normalized by $\nu$ so that the mean of each distribution is 1 , and the scale of the ordinate is arbitrary.

\subsection{Application to Shallow Water Surface Gravity Waves}

Using bispectral analysis, Elgar and Guza [1985, p. 432] concluded that narrow-band swell (peak period about $16 \mathrm{~s}$ ) observed in $9 \mathrm{~m}$ water depth was ". . . qualitatively consistent with Stokes-like nonlinearities." Their conclusion was based on observed values of biphase for triads consisting of the power spectral primary peak frequency $\left(f_{p}\right)$ and its first few harmonics. Based on the results presented above, bispectral estimates from these data can now be interpreted quantitatively. For dof $=256$ the observed values of bicoherence and biphase for the two lowest-order triads were $\hat{b}^{2}\left(f_{p}, f_{p}\right)=0.161, \hat{\beta}\left(f_{p}, f_{p}\right)=-7^{\circ}$ and $\hat{b}^{2}\left(f_{p}, f_{2 p}\right)=0.067$, $\hat{\beta}\left(f_{p}, f_{2 p}\right)=-35^{\circ}$. (Bicoherences at other triads were not significantly greater than zero.) Accounting for the bias, equation (4), $b_{\text {mele }}^{2}\left(f_{p}, f_{p}\right)=0.160$ and $b_{\text {mele }}^{2}\left(f_{p}, f_{2 p}\right)=0.070$.

For dof $=256$ and $b_{\text {mele }}^{2}$ as given above, $90 \%$ confidence limits for biphase can be constructed. For the self-self interaction $\left(f_{p}, f_{p}, f_{2 p}\right)$ the $90 \%$ limits are $\pm 13.5^{\circ}$. Thus the observed value $\left(-7^{\circ}\right)$ is within the $90 \%$ limits of the Stokes biphase, $\beta=0^{\circ}$, and the data are consistent with Stokes-like nonlinearities. On the other hand, $90 \%$ confidence limits for the biphase of the triad $\left(f_{p}, f_{2 p}, f_{3 p}\right)$ are $\pm 21.6^{\circ}$ and do not include the observed value $\left(-35^{\circ}\right)$. Consequently, with a $10 \%$ possibility of being incorrect, it is concluded that the biphase of this triad is not consistent with Stokes-like nonlinearities and that shallow water resonance effects [Freilich and Guza, 1984] are important.

\section{Conclusions}

Numerical simulations of random processes with nonzero bicoherence were used to investigate the statistics of estimates of bicoherence and biphase. Bicoherence is biased (Figure 1) and is approximately $\alpha \chi^{2}$ distributed (Figure 3), with parameter $\nu$ a function of the true bicoherence and the degrees of freedom associated with the estimate (equation (8)). Confidence limits for estimates of bicoherence can be constructed from the $\alpha \chi^{2}$ distribution (i.e., from $b^{2}$ and dof) and agree with the corresponding values observed in the simulations (Figure 4). The statistics of biphase are similar to those of the phase of the cross spectrum (equation (9) and Figure 5), and confidence limits for biphase estimates based on $b^{2}$ and dof also agree with corresponding values observed in the simulations (Figure 6). Maximum likelihood estimates of the true value of bicoherence given an observed value were obtained from the $\alpha \chi^{2}$ distribution (Table 1 and Figure 7). The ratio of maximum likelihood estimates of bicoherence to observed values approaches 1 as $\hat{b}^{2}$ and/or dof increases, with less than $10 \%$ differences if $b^{2}>0.25$ (for dof $\geq 32$ ) and/or dof $>150$ (for $\hat{b}^{2} \geq 0.1$ ) (Figure 8).

Acknowledgments. This research was supported by the Coastal Sciences Branch of the Office of Naval Research and by the Physical Oceanography Program of the National Science Foundation. The computations were performed at the San Diego Supercomputer Center (funded by NSF). R. T. Guza made helpful comments.

\section{REFERENCES}

Abramowitz, M., and I. Stegun, Handbook of Mathematical Functions, p. 1046, Dover, New York, 1972

Ashley, R. A., D. M. Patterson, and M. J. Hinich, A diagnostic test for nonlinear serial dependence in time series fitting errors, $J$. Time Ser. Anal., 7, 165-178, 1986.

Beningus, V. A., Estimation of the coherence spectrum and its confidence interval using the fast Fourier transform, IEEE Trans. Audio Electroacoust., 17, 145-150, 1969.

Brillinger, D. R., An introduction to polyspectra, Ann. Math. Stat., 36, 1351-1374, 1965.

Brillinger, D. R., and M. Rosenblatt, Asymptotic theory of estimates of $k$-th order spectra, in Advanced Seminar on Spectral Analysis of Time Series, edited by B. Harris, pp. 153-188, John Wiley, New York, $1967 a$.

Brillinger, D. R., and M. Rosenblatt, Computation and interpretation of $k$-th order spectra, in Advanced Seminar on Spectral Analysis of Time Series, edited by B. Harris, pp. 189-232, John Wiley, New York, $1967 b$.

Doering, J. C., and A. J. Bowen, Skewness in the nearshore zone: A comparison of estimates from Marsh-McBirney current meters and collocated pressure sensors, J. Geophys. Res., 92, 13,17313,183, 1987.

Elgar, S., and R. T. Guza, Observations of bispectra of shoaling surface gravity waves, J. Fluid Mech., 161, 425-448, 1985.

Elgar, S., and R. T. Guza, Nonlinear model predictions of bispectra of shoaling surface gravity waves, $J$. Fluid Mech., 167, 1-18, 1986.

Elgar, S., and R. T. Guza, Statistics of bicoherence, IEEE Acoust. Speech Signal Process., 36, 1667-1668, 1988.

Elgar, S., R. T. Guza, and R. J. Seymour, Wave group statistics from numerical simulations of a random sea, Appl. Ocean. Res. 7, 93-96, 1985.

Freilich, M. H., and R. T. Guza, Nonlinear effects on shoaling surface gravity waves, Philos. Trans. R. Soc. London, Ser. A $311,1-41,1984$

Freilich, M. H., and S. S. Pawka, Statistics of $S_{x y}$ estimates, $J$. Phys. Oceanogr., 17, 1786-1797, 1987.

Hasselman, K., W. Munk, and G. MacDonald, Bispectra of ocean waves, in Time Series Analysis, edited by M. Rosenblatt, pp. 125-139, John Wiley, New York, 1963.

Haubrich, R. A., Earth noises, 5 to 500 millicycles per second, $1, J$. Geophys. Res., 70, 1415-1427, 1965.

Hinich, M. J., Testing for Gaussianity and linearity of a stationary time series, J. Time Ser. Anal., 3, 66-74, 1982.

Hinich, M. J., and C. S. Clay, The application of the discrete Fourier transform in the estimation of power spectra, coherence, and bispectra of geophysical data, Rev. Geophys., 6, 347-363, 1968.

Jenkins, G. M., and D. G. Watts, Spectral Analysis and Its Applications, Holden-Day, San Francisco, Calif., 1968.

Kim, Y. C., and E. J. Powers, Digital bispectral analysis and its application to nonlinear wave interactions, IEEE Trans. Plasma Sci., 1, 120-131, 1979. 
McComas, C. H., and M. G. Briscoe, Bispectra of internal waves, J. Fluid Mech., 97, 205-213, 1980.

Müller, D., Bispectra of sea-surface temperature anomalies, $J$. Phys. Oceanogr., 17, 26-36, 1987.

Neshyba, S., and E. J. C. Sobey, Vertical cross coherence and cross bispectra between internal waves measured in a multiple layered ocean, J. Geophys. Res., 80, 1152-1162, 1975.

Nikias, C. L., and M. R. Raghuveer, Bispectrum estimation: A digital signal processing framework, Proc. IEEE, 75, 867-891, 1987.

Roden, G. I., and D. J. Bendiner, Bispectra and cross-bispectra of temperature, salinity, sound velocity, and density fluctuations with depth off northeastern Japan, J. Phys. Oceanogr., 3, 308317, 1973.

Rosenblatt, M., and J. W. Van Ness, Estimation of the bispectrum, Ann. Math. Stat., 36, 1120-1136, 1965.

S. Elgar and G. Sebert, Electrical and Computer Engineering Department, Washington State University, Pullman, WA 991642752.

(Received December 20, 1988; accepted March 27, 1989.) 